\title{
ORDERS IN SIMPLE LIE ALGEBRAS OF CHEVALLEY TYPE
}

\author{
JAMES F. HURLEY
}

(Received 29 March 1977)

Communicated by $\mathrm{H}$. Lausch

\begin{abstract}
The integral structure of a simple Lie algebra $L$ of Chevalley type over a field $F$ of fractions of an integral domain $D$ is studied. Sandwich relations for sufficiently large orders are obtained, including a new general sandwich relation for orders of $L$ in case $D$ is an integrally closed Noetherian domain. Generalizations of the principal results of Hyman (1966) in the case when $D$ is a ring of algebraic integers are obtained, using techniques developed by the author and Stewart (1973) which are applied to certain orders in $L$ that arise in a natural fashion from the Chevalley basis.
\end{abstract}

Subject classification (Amer. Math. Soc. (MOS) 1970): primary 17 B 20; secondary 17 B 10, 17 B 45, 20 G 15, 20 G 05.

\section{Introduction}

Since Chevalley (1955) showed for a simple finite dimensional Lie algebra $L$ over the complex field the existence of a basis relative to which the structure constants are integers, the arithmetic theory of Lie algebras has developed as an area of considerable attention. In addition to playing a fundamental role in the construction of finite simple groups, Chevalley bases have been a prominent part of much recent work in algebraic group theory. To mention a few examples, Springer (1966) has used the arithmetic of semisimple Lie algebras to obtain (under mild restrictions on $p$ ) the existence of regular unipotent elements in semisimple algebraic groups over algebraically closed fields of characteristic $p$ (see also Steinberg (1965)), and the ideal arithmetic of simple Lie algebras has been used by the author (1971) to construct normal subgroups of certain algebraic groups over rings and by Abe (1969) and Abe-Suzuki (1976) to classify the normal structure of Chevalley groups over local rings and Dedekind domains.

The present paper explores the integral structure of a simple Lie algebra $L$ of Chevalley type over a field $F$ which is the field of fractions of an integral domain $D$. To study forms of $L$ over $D$ (that is, algebras $A$ over $D$ such that $F \otimes_{D} A \cong L$ ) it suffices to study orders in $L$. Using to good advantage results of Stewart (1973) we obtain sandwich relations for sufficiently large orders in $L$ involving the orders which arise naturally from the Chevalley basis for $L$ (Theorem 1). Then application of methods in the spirit of an earlier paper (1969) lets us obtain in rather direct 
fashion generalizations of the principal results of the (otherwise unpublished) doctoral dissertation of M. Harvey Hyman (1966) (Theorems 2 and 4). Finally, Stewart's results in combination with ours (1971) are used to obtain a new general sandwich relation for orders in $L$ in case $D$ is an integrally closed Noetherian integral domain (Theorem 3).

\section{Background}

In this section definitions of the main ideas of the present paper are given, as well as some preliminary results which do not seem to be in the literature apart from special cases in Hyman (1966).

Let $D$ be an integral domain not of characteristic 2 or 3 . In case we are dealing with a Lie algebra of type $A_{n}$, we further assume that $n+1$ is not a multiple of the characteristic of $D$. Let $F$ be the field of fractions of $D$, and $V$ a finite dimensional vector space over $F$. We assume $F \neq D$, that is, $D$ is not a field.

Definition 1 . A finitely generated $D$-module $\mathscr{L} \subseteq V$ which spans $V$ over $F$ is called a lattice in $V$.

DefinITION 2. If $L$ is a finite dimensional Lie algebra over the field $F$, then a lattice $O \subseteq L$ is an order in $L$ if it is closed under multiplication. (In a natural way then we can regard $\mathcal{O}$ as a Lie algebra over the ring $D$.)

Henceforth we assume that $L$ is a finite dimensional split (Jacobson, 1962) simple Lie algebra over $F$, and $H$ is a Cartan subalgebra. Then $L$ has a basis $B=\left\{e_{r} \mid r \neq 0\right\} \cup\left\{h_{1}, h_{2}, \ldots, h_{n}\right\}$ made up of root vectors $e_{r}$ and a basis for $H$ satisfying

$\left[e_{r}, e_{-r}\right]=h_{r}$, an integral linear combination of the $h_{i}$,

$\left[h_{i}, h_{j}\right]=0$,

$\left[e_{r}, e_{s}\right]= \pm N_{r s} e_{r+s}$ if $r+s \neq 0$, where $N_{r s}$ is 0 in case $r+s$ is not a root and is $p_{r s}+1$ otherwise, $p_{r g}$ being the greatest integer $i$ such that $s-i r$ is a root,

$\left[h_{r}, e_{s}\right]=c(s, r) e_{s}$, where $c(s, r)=2(s, r) /(r, r)$ is the Cartan integer of $s$ and $r$ (see Chevalley, 1955).

The Chevalley order $L_{D}$ is the $D$-submodule of $L$ generated by the Chevalley basis $B$. For a more complete discussion of Chevalley bases, see Chevalley (1955), Steinberg (1968) or Hurley (1969). An algebra $L$ with such a basis $B$ is said to be of Chevalley type.

LEMMA 1. If $D$ is a Dedekind domain and $T$ is a linear transformation of $L$ into itself which leaves an order $\mathcal{O}$ invariant, then Trace $T \in D$ and det $T \in D$.

Proof. Extend $F$ to a field $K$ which contains all the eigenvalues of $T$. Let $\bar{D}_{K}$ be the integral closure of $D$ in $K$. Consider the extensions $L_{K}=K \otimes_{F} L$, $T_{K}=1 \otimes_{F} T$, and $\mathcal{O}_{K}$, the $\bar{D}_{K}$ module generated by $1 \otimes_{F} \mathcal{O}$. We have $T_{K}: \mathcal{O}_{K} \rightarrow \mathcal{O}_{K}$, 
and $T_{K}$ has the same eigenvalues as $T$. Let $x \in L_{K}$ be an eigenvector belonging to any eigenvalue $\lambda$. Now $\mathcal{O}_{K} \cap K x$ cannot be 0 since $x \in L_{K}$, the $K$-span of $\mathcal{O}_{K}$. Thus $\mathcal{O}_{K} \cap K x$ is a torsion free finitely generated $\bar{D}_{K}$-module. Also,

$$
T_{K}\left(\mathcal{O}_{K} \cap K x\right)=\lambda \mathscr{O}_{K} \cap K x \subseteq \mathcal{O}_{K} \cap K x,
$$

hence by Lemma 22.4 of Curtis-Reiner $\left(1962\right.$, p. 146) $\lambda \in \bar{D}_{K}$ since $\bar{D}_{K}$ is a Dedekind domain by Zariski-Samuel $(1958$, p. 281). Thus $\lambda$ is integral over $D$. So all eigenvalues of $T$ are integral over $D$. Then all the coefficients of the characteristic polynomial of $T$ (which are sums of products of the eigenvalues) are integral over $D$ by Zariski-Samuel $(1958$, p. 255$)$ and are in $F$. Since $D$ is a Dedekind domain, it is integrally closed (that is, integrally closed in $F$ ), so all the coefficients of the characteristic polynomial of $T$ are actually in $D$. In particular then, Trace $T \in D$ and $\operatorname{det} T \in D$.

Proposition 1. If $D$ is a Dedekind domain, then $L$ has infinitely many orders.

Proof. Consider a nonzero element $a \in D$ which is not invertible. Then $a, a^{2}, a^{3}, \ldots$ is a sequence of nonzero elements of $D$ which are not invertible. We then have $a^{n} L_{D} \supset a^{n+1} L_{D}$. For inclusion is clear, and $a^{n+1} L_{D}=T\left(a^{n} L_{D}\right)$ where

$$
T: a^{n} L_{D} \rightarrow a^{n} L_{D}
$$

is given by $T(x)=a x$. Since $\operatorname{det} T=a^{n}$ is not invertible in $D, T \notin G L\left(a^{n} L_{D}\right)$ in view of Lemma 1 above. For if $T^{-1} \in G L\left(a^{n} L_{D}\right)$, then $\operatorname{det} T^{-1}=(\operatorname{det} T)^{-1}=\left(a^{n}\right)^{-1}$ would be in $D$. Thus $T$ is not onto, so the inclusion is proper. We then have an infinite descending chain of orders $L_{D} \supset a L_{D} \supset a^{2} L_{D} \supset a^{3} L_{D} \supset \ldots$ in $L$.

This result was obtained by Hyman $(1966$, p. 25) for the case of $D$ a ring of algebraic integers. We now focus attention on the Chevalley order $L_{D}$. In many cases the orders which contain this order will comprise a manageable collection.

Definition 3. The superstructure of an order $\mathcal{O}$ is the collection of orders in $L$ which contain $\mathcal{O}$.

For Dedekind domains we can show that the superstructure of an order in $L$ is relatively simple.

Proposition 2. Let $D$ be a Dedekind domain of characteristic 0 or $p$ where $p \nmid n+1$ if $L$ is of type $A_{n}$ or $C_{n}, p \nmid 2 n-1$ if $L$ is of type $B_{n}, p \nmid n-1$ if $L$ is of type $D_{n}$, and $p \neq 5$ if $L$ is of type $E_{8}$. Let $\mathscr{L}$ be a lattice in $L$. Then every order $\mathcal{O}$ containing $\mathscr{L}$ is contained in a fixed lattice $\mathscr{L}^{*}$.

Proof. Since the Killing form $K$ is nondegenerate on $L$ (Jacobson, 1962, p. 70), for any basis $B=\left\{v_{1}, v_{2}, \ldots, v_{r}\right\}$ of $L$ made up of elements of $\mathscr{L}$, there is a dual basis $B^{*}=\left\{v_{1}^{*}, v_{2}^{*}, \ldots, v_{r}^{*}\right\}$ such that $K\left(v_{i}, v_{j}^{*}\right)=\delta_{i j}$ for $i, j=1,2, \ldots, r$. Let 
$\mathscr{L}^{*}=\sum_{i=1}^{r} D v_{i}^{*}$ and let $\mathcal{O}$ be any order in $L$ such that $\mathcal{O} \supseteq \mathscr{L}$. For $x \in \mathcal{O}$, write $x=\sum_{i=1}^{r} d_{i} v_{i}^{*}, d_{i} \in F$. Then $K\left(x, v_{j}\right)=K\left(\sum_{i=1}^{r} d_{i} v_{i}^{*}, v_{j}\right)=d_{j}$. Now $x \in \mathcal{O}$, and so we have ad $x: \mathcal{O} \rightarrow \mathcal{O}$, and ad $v_{j}: \mathcal{O} \rightarrow \mathcal{O}$ since $v_{j} \in \mathscr{L} \subseteq \mathcal{O}$. Thus ad $x_{\circ}$ ad $v_{j}: \mathcal{O} \rightarrow \mathcal{O}$, and $\operatorname{ad} x_{0} \operatorname{ad} v_{j}$ is a linear transformation of $L$. So by Lemma 1 ,

$$
\text { Trace }\left(\operatorname{ad} x \circ \operatorname{ad} v_{j}\right)=K\left(x, v_{j}\right)=d_{j} \in D .
$$

Thus $x=\sum_{i=1}^{r} d_{i} v_{i}^{*} \in \mathscr{L}^{*}$ and we have $\mathscr{L} \subseteq \mathcal{O} \subseteq \mathscr{L}^{*}$.

Proposition 2 holds in particular if $\mathscr{L}$ is an order $\mathcal{O}$. If $D$ is a ring of algebraic integers in a finite extension $K$ of $Q$, then Hyman $(1966, p .21)$ showed the superstructure of such an $\mathcal{O}$ is actually finite.

Hyman's dissertation consisted mainly of a study of the superstructure of $L_{D}$ in case $D$ was the ring of algebraic integers of a finite extension of the rational field (hence $D$ was a Dedekind domain). His first theorem concerned the algebra of type $A_{1}$, in which case the superstructure of $L_{D}$ consists of five orders. These are $L_{D}$ (with basis $\{e, h, f\}$ ), $L_{D}^{\prime}$ (with basis $\left\{e, \frac{1}{2} h, f\right\}$ ) in which $L_{D}$ has index 2 , and three orders $\mathscr{K}_{1}, \mathscr{H}_{2}, \mathscr{H}_{3}$, in which $L_{D}^{\prime}$ has index 2 . $\mathscr{M}_{1}$ has basis $\left\{e, \frac{1}{2} h, \frac{1}{2} f\right\} ; \mathscr{M}_{2}$ has basis $\left\{\frac{1}{2} e, \frac{1}{2} h, f\right\}$; and $\mathscr{H}_{3}$ has basis $\left\{e, \frac{1}{2}(e+h),-\frac{1}{2} e-\frac{1}{2} h+\frac{1}{2} f\right\}$. For a proof, see Hyman (1966, pp. 67-69). Theorems 2 and 4 below generalize the remaining two theorems of Hyman (1966) and are proved in a more direct manner here.

\section{A sandwich relation for orders}

Our first theorem gives upper and lower bounds for an order containing the Chevalley order $L_{D}$.

THEOREM 1. Let $\mathcal{O}$ be an order in $L$ which contains $L_{D}$. Then there is an integer $k$ such that

$$
k J L_{D} \subseteq \mathcal{O} \subseteq J L_{D}
$$

where $J$ is the smallest $D$-submodule of $F$ such that $J L_{D}$ contains $\mathcal{O}$. The prime factors of $k$ are in the set $\left\{2,3, p_{1}, \ldots, p_{m}\right\}$, where the $p_{i}$ are the prime divisors of $\operatorname{det} C$, the determinant of the Cartan matrix $C$ of $L$.

Proof. First, $J$ is well defined. We have in fact $O \subseteq J^{\prime} L_{D}$, if $J^{\prime}$ is the $D$-submodule of $F$ generated by 1 and all coefficients $c_{r}, c_{i}$ of the elements $x$ of $\mathcal{O}$ expressed uniquely as $F$-linear combinations of the members of $B, x=\sum_{i=1}^{n} c_{i} h_{i}+\sum_{r} c_{r} e_{r}$. Next we observe that $D$ satisfies the hypotheses for the ground ring in Theorem 3.1 of Stewart (1973). Then as in the proof of that result, for an appropriate integer $k$ there is an element of the multiplication ring of $L_{D}$ sending $x$ to $k c_{r} e_{s}$ and another sending $x$ to $k c_{j} h_{i}$, for arbitrary roots $r$ and $s$ and arbitrary positive integers $i$ and $j$. For if we represent an arbitrary element of $B$ by the generic symbol $p_{\alpha}$, then there is a $v_{\alpha \beta}$ in the multiplication ring of $L_{\mathrm{Z}}$ sending $p_{\alpha}$ to $k \delta_{\alpha \beta} p_{\beta}$. If $v_{\alpha \beta}$ is expressed as a sum of products of left and right multiplications by elements $y$ in $L_{Z}$, then 
$\bar{v}_{\alpha \beta}$ sends $\sum d_{\alpha} \otimes p_{\alpha}$ to $k \delta_{\alpha \beta} d_{\alpha} \otimes p_{\beta}$, where $\bar{v}_{\alpha \beta}$ is the corresponding sum of products of left and right multiplications by elements $1 \otimes y$ in $L_{D}$. Since $\mathcal{O} \supseteq L_{D}$, all the $k c_{r} e_{s}$ and $k c_{j} h_{i}$ belong to $\mathcal{O}$. Hence $\mathcal{O} \supseteq k J L_{D}$. The proof is then complete as soon as we remark that $k$ has only the factors specified by Proposition 4.1 of Stewart (1973).

If $D$ is a Noetherian integral domain (in particular a Dedekind domain), then we remark that $J$ in Theorem 1 is actually a fractional ideal (Curtis-Reiner, 1962, p. 107). For $\mathcal{O}$ is a finitely generated $D$-module (since it is an order) and $k J L_{D}$ is a submodule, hence also finitely generated if $D$ is Noetherian. Thus $J L_{D}$ is a finitely generated $D$-module. But then $J$ is a finitely generated $D$-module (or else no finite number of elements of $J$ would suffice to generate the coefficients of elements in $J L_{D}$ ), that is, $J$ is a fractional ideal.

\section{Superstructure of the Chevalley order}

We now proceed to a detailed study of the orders $\mathcal{O}$ in $L$ which contain the Chevalley order $L_{D}$.

Definition 4. If $D$ is an integral domain with quotient field $F$, then $P$ represents the lattice of weights of all representations of $L_{F} . P$ has as $\mathrm{Z}$-basis the fundamental weights $\left\{w_{1}, w_{2}, \ldots, w_{n}\right\}$ given by $w_{i}\left(h_{j}\right)=\delta_{i j}$. The lattice of $D$-coweights is ${ }_{D} P^{\perp}=\{h \in H \mid w(h) \in D$ for all $w \in P\}$, which has basis $\left\{h_{1}, h_{2}, \ldots, h_{n}\right\}$ over $D$. This coincides with $H_{D}$, the abelian algebra $D \otimes_{\mathrm{z}} H_{\mathrm{z}}$, and we shall use the latter notation frequently.

DefinITION 5. $P_{r}$ represents the free abelian group generated by the roots of $L$ relative to $H . P_{r}$ has $\mathrm{Z}$-basis $\left\{r_{1}, r_{2}, \ldots, r_{n}\right\}$, the set of simple roots. Recall $r_{i}=\sum_{j=1}^{n} c\left(r_{i}, r_{j}\right) w_{j}$. The lattice of $D$-coroots is

$$
{ }_{D} P_{r}^{\perp}=\{h \in H \mid r(h) \in D \text { for every root } r\} \text {. }
$$

We also need the lattice of $\bar{D}$-coroots defined by replacing $D$ by $\bar{D}$ in ${ }_{D} P_{r}^{\perp}$ where $\bar{D}$ is the integral closure of $D$ (that is, the integral closure of $D$ in $F$, so that $\bar{D}=D$ if $D$ is integrally closed). The lattice ${ }_{D} P_{r}^{\perp}$ is labelled $H_{D}^{\prime}$ in Hurley $(1969,1971)$. $L_{D}^{\prime}$ is the algebra $D \otimes_{\mathrm{Z}} L_{\mathrm{Z}}^{\prime}$ where $L_{\mathrm{Z}}^{\prime}=E_{\mathrm{Z}} \oplus H_{\mathrm{Z}}^{\prime}, E_{\mathrm{Z}}$ the free abelian group on the root vectors $e_{r}$.

Lemma 2. Suppose that $\alpha \in F$ and the coweight $h_{r} \in H_{D}$. Then $\alpha h_{r} \in H_{D}$ if and only if $\alpha \in D$.

Proof. If $a \in D$, then for any weight $w \in P$, we have $w\left(\alpha h_{\tau}\right)=\alpha w\left(h_{r}\right) \in D$, since $h_{r} \in H_{D}={ }_{D} P^{\perp}$. Conversely, if $\alpha h_{r} \in H_{D}$, then we can express $\alpha h_{r}$ in terms of the basis elements $h_{i}: \alpha h_{r}=\sum_{i=1}^{n} c_{i} h_{i}, c_{i} \in D$. Find $w \in P$ such that $w\left(h_{r}\right)=1$. (The existence of $w$ follows from the fact that $r$ belongs to some simple system of roots, 
so has a corresponding fundamental weight $w$, or from Jacobson $(1962$, p. 140).) Then apply $w$ to $\alpha h_{r}$. We get $\alpha \cdot 1=\sum_{i=1}^{n} c_{i} w\left(h_{i}\right)$, and $w\left(h_{i}\right) \in D$ since $w \in P$, $h_{i} \in H_{D}={ }_{D} P^{\perp}$. Thus $\alpha \in D$.

Lemma 3. Suppose that $\alpha \in F$ and $r$ is a root. Then

(1) If $L$ is not of type $B_{n}(n \geqslant 2)$ or $A_{1}$, then we have $\alpha h_{r} \in_{D} P_{r}^{\perp}$ if and only if $\alpha \in D$.

(2) If $L$ is of type $B_{n}$, then for a long root $r, \alpha h_{r} \in_{D} P_{r}^{\perp}$ if and only if $\alpha \in D$, while for a short root $r, \alpha h_{r} \in{ }_{D} P_{r}^{\perp}$ if and only if $2 \alpha \in D$.

(3) If $L$ is of type $A_{1}$, then $\alpha h_{r} \in_{D} P_{r}^{\perp}$ if and only if $2 \alpha \in D$.

The same assertions hold if $D$ is replaced by $\bar{D}$.

Proor. We have $\alpha h_{r} \in{ }_{D} P_{r}^{\perp}$ if and only if $s\left(\alpha h_{r}\right) \in D$ for all roots $s$, that is, if and only if $\alpha c(s, r) \in D$ for all roots $s$. Suppose first that $\alpha h_{r} \in{ }_{D} P_{r}^{\perp}$. In the single root length case, find a root $s$ such that $s+r$ is a root. We then have $c(s, r)=-1$, so $\alpha \in D$. In the general case we can imbed $r$ into a simple system of roots. Then all the $c(s, r)$ as $s$ varies over this simple system occur in some fixed column of the Cartan matrix. In all cases save $A_{1}, G_{2}$, and $B_{n}$, at least one entry in any column of the Cartan matrix is -1 , so choosing corresponding $s$ we get $\alpha c(s, r)=-\alpha \in D$. In type $G_{2}$, the Cartan matrix is $\left(\begin{array}{cc}2 & -1 \\ -3 & 2\end{array}\right)$ and so if $r=r_{1}$, then we have $2 \alpha$ and $-3 \alpha$ in $D$ and hence $\alpha=-2 \alpha+3 \alpha \in D$. (If $r=r_{2}$, then the previous reasoning gives $\alpha \in D$.) In type $B_{n}$, every column has a -1 except for the last column, which corresponds to the Cartan integers $c(s, r)$ for the short simple root $r$. This means that if $r$ is short, then we can only conclude $2 \alpha \in D$, and if $r$ is long, then as before $\alpha \in D$. The proof of this half is now complete as soon as we remark that in type $A_{1}$ the Cartan matrix is (2). For the converse, if $\alpha \in D$, then clearly $\alpha h_{r} \in{ }_{D} P_{r}^{\perp}$. In type $B_{n}$ with $r$ short, if $2 \alpha \in D$, then again imbed $r$ into a simple system of roots. Since $c(s, r)$ is linear in $s$, it is sufficient to consider $s$ ranging over this simple system. For either simple root $s$ not orthogonal to $r$, we have $s\left(\alpha h_{r}\right)=\alpha c(s, r)= \pm 2 \alpha \in D$. The case $A_{1}$ is clear. Finally, it is apparent that $D$ can be replaced throughout by $\bar{D}$.

Lemma 4. Suppose that $D$ is a Noetherian integral domain. Let $h \in H$ belong to an order $\mathcal{O}$ in $L$. Then $h \in_{\bar{D}} P_{r}^{\perp}$, that is, $h$ is a $\bar{D}$-coroot.

Proof. Consider the linear transformation ad $h: L \rightarrow L$. Then ad $h$ leaves $\mathcal{O}$ invariant. Since $L$ is split, the eigenvalues of ad $h$ all belong to $F$, and are the values $r(h)$ for $r$ a root of $L$ relative to $H$. Then as in the proof of Lemma 1, with $F$ playing the role of $K$, we see that all the eigenvalues of $\operatorname{ad} h$ are integral over $D$. Thus $r(h) \in \bar{D}$ for all roots $r$, that is, $h$ is a $\bar{D}$-coroot.

We now have all the tools we need to generalize the main result of Hyman (1966) on the superstructure of $L_{D}$. 
THEOREM 2. Let $L$ be a simple Lie algebra of Chevalley type and rank at least 2 over $F$, the field of fractions of the Noetherian integral domain $D$. Let $\mathcal{O}$ be an order in $L$ which contains the Chevalley order $L_{D}$. In case $L$ is of type $B_{n}$ or $C_{n}$, assume additionally that 2 is invertible in $D$. Then $L_{D} \subseteq \mathcal{O} \subseteq L_{\bar{D}}$, where $\bar{D}$ is the integral closure of $D$.

Proof. In the non-symplectic cases, 5.1, 5.2, and 5.3 of Hurley (1969) show that given any $x=\Sigma c_{r} e_{r}+h \in \mathcal{O}$, since each $e_{r} \in \mathcal{O}$ we can obtain each $c_{r} e_{r} \in \mathcal{O}$ by multiplication by suitable root vectors $e_{s}$. Thus $\mathcal{O} \cap \mathrm{Fe}_{r}$ is a direct summand of $\mathcal{O}$. Now let $A_{r}=\left\{a \in F \mid a e_{r} \in \mathcal{O} \cap F e_{r}\right\}$. Then $A_{r}$ is a $D$-submodule of $F$. The map $a \mapsto a e_{r}$ establishes a $D$-module isomorphism between $A_{r}$ and $\mathcal{O} \cap F_{r_{r}}$. Since $\mathcal{O}$ is a finitely generated $D$-module by definition, its direct summand $O \cap F e_{r}$ is also finitely generated. Thus $A_{r}$ is a finitely generated $D$-module, and thus is a fractional ideal. Then by Lemma $4, r(h) \in \bar{D}$. So $h \in L_{\bar{D}}^{\prime}$. Also we have from $c_{r} e_{r} \in \mathcal{O}$ that $\left[c_{r} e_{r}, e_{-r}\right]=c_{r} h_{r} \in \mathcal{O}$. So by Lemma $4, c_{r} h_{r} \in \bar{D}_{D} P_{r}^{\perp}$. Hence by Lemma 3 and our assumption about $2, c_{r} \in \bar{D}$. It now follows that $x=h+\sum c_{r} e_{r} \in L_{\bar{D}}^{\prime}$.

Some restriction on 2 seems essential in the case of $B_{n}$ even if $D$ is a Dedekind domain. For example, see Hyman (1966, p. 115) where the restriction that 2 be unramified in $F$ (recall that $D$ is a ring of algebraic integers in that paper) allows the theorem just proved to go through.

In case $C_{n}$ if we remove the assumption that 2 is invertible, then we can only conclude from $x=h+\sum c_{r} e_{r} \in \mathcal{O}$ that $c_{s} e_{s} \in \mathcal{O}$ for every short root $s$, and $2 c_{t} e_{t} \in \mathcal{O}$ for every long root $t$. While the submodules $A_{r}$ and $2 A_{s}$ are finitely generated since they are isomorphic to $D$-submodules $O \cap F e_{r}$ and $2 O \cap \cap F e_{s}$ respectively of the finitely generated module $\mathcal{O}$, we cannot conclude that $h \in \mathcal{O}$ and hence that $h \in L_{\bar{D}}^{\prime}$. Hyman's less direct approach does allow this difficulty to be overcome if $D$ is a ring of algebraic integers. See Hyman (1966, pp. 114-117).

Corollary. Let $D$ be of rank at least 2 . Let $D$ be a Noetherian integrally closed integral domain such that in type $B_{n}$ and $C_{n} 2$ is invertible in $D$. Then for each order $\mathcal{O} \supseteq L_{D}$, there is a unique $D$-module $M$ lying between the lattice $H_{D}={ }_{D} P^{\perp}$ and the lattice $H_{D}^{\prime}={ }_{D} P_{r}^{\perp}$ of coroots such that $\mathcal{O}=M \oplus\left(\mathcal{O} \cap E_{D}\right)$.

Proof. In this case $D=\bar{D}$ and so $L_{\bar{D}}^{\prime}=L_{D}^{\prime}=E_{D} \oplus H_{D}^{\prime}=E_{D}{ }_{D} P \frac{\perp}{r}$. As the proof of Theorem 2 shows, $M=H_{D} \cap \mathcal{O}$ is a direct summand of $\mathcal{O}$ and is contained in $H_{D}^{\prime}={ }_{D} P_{r}^{\frac{1}{r}}$. Since $\mathcal{O} \supseteq L_{D}, M \supseteq H_{D}$.

As a special case, we obtain Theorem 3 of Hyman (1966) in the non-symplectic cases, and in all cases if 2 is invertible in $D$. 
THEOREM 3. Let $L$ be of rank at least 2, $D$ a Noetherian integrally closed integral domain, and $\mathcal{O} \supseteq L_{D}$ an order in $L$. Then $k L_{D}^{\prime} \subseteq \mathcal{O} \subseteq L_{D}^{\prime}$ for some integer $k$, assuming again that $2 \in D$ is invertible in case $L$ is of type $B_{n}$ or $C_{n}$.

ProOr. Such an order $\mathcal{O}$ is contained, in view of the Corollary to Theorem 2, in $L_{D}^{\prime}$, and so is an algebra lying between $L_{D}$ and $L_{D}^{\prime}$. Then by 2.6 of Hurley (1971), $\mathcal{O}$ is an ideal in $L_{D}^{\prime}$ whose intersection with $E_{D}$ is $E_{D}$. As $L_{D}^{\prime}=D \otimes_{Z} L_{Z}^{\prime}$ and $D$ is commutative with identity, we can apply Theorem 3.1 of Stewart (1973) to conclude that there is an integer $k$ such that $k J L_{D}^{\prime} \subseteq \mathcal{O} \subseteq J L_{D}^{\prime}$ for the ideal $J$ of $D$ generated by the coefficients of the elements of $\mathcal{O}$. Since $\mathcal{O} \supseteq E_{D}, J=(1)=D$. Thus $k L_{D}^{\prime} \subseteq \mathcal{O} \subseteq L_{D}^{\prime}$.

From this and Proposition 4.1 of Stewart (1973), we have the following information about the integer $k$.

Corollary. The integer $k \leqslant \Pi p_{i}$ where $\left\{p_{i}\right\}$ is the set of prime divisors of det $C$ and $m$, the ratio of the squares of the lengths of the long to the short roots.

\section{Integral orders}

We now proceed to study integral representations of $L$.

Definition 6. Let $\mathcal{O}$ be an order in $L$. Let $\pi: L \rightarrow$ End $V$ be a representation of $L$ on the finite dimensional vector space $V$ over $F$. $\mathcal{O}$ is $\pi$-integral if $\pi(\mathcal{O}) \subseteq$ End $\mathscr{L}$ for some lattice $L$ in $V . \mathcal{O}$ is called an integral order in case $\mathcal{O}$ is $\pi$-integral for every representation $\pi$ of $L$ over $F$. An element $x$ in $L$ is $\pi$-integral if $\pi(x) \in$ End $\mathscr{L}$ for some lattice $\mathscr{L} \subseteq V$. The element $x \in L$ is an integral element if it is $\pi$-integral for every representation $\pi$ of $L$ over $F$.

Lemma 5. If $h \in H$ is integral and $D$ is a Noetherian integral domain, then $w_{i}(h) \in \bar{D}$ for every fundamental weight $w_{i}$, and so $h \in H_{\bar{D}}=\bar{D}_{D} P^{\perp}$.

ProOF. If $h$ is integral, then for any representation $\pi \pi(h)$ is an integral element. In particular if $\pi$ is a representation with $w_{i}$ as a weight, then $\pi(h)$ has eigenvalue $w_{i}(h) \in F$ since $\pi(h) v=w_{i}(h) v$, for some weight vector $v$ belonging to $w_{i}$. If $\mathscr{L}$ is a lattice in $V$ such that $\pi(h) \in$ End $\mathscr{L}$, then $\mathscr{L} \cap F v \subseteq \mathscr{L}$ is a finitely generated $D$-module of the form $A v$ where $A=\{a \in F \mid a v \in \mathscr{L}\}$ is finitely generated, as in the proof of Theorem 2. Now $\pi(h) \mathscr{L} \cap F v \subseteq \mathscr{L} \cap F v$ and so $w_{i}(h) A \subseteq A$. Then $w_{i}(h)^{m} A \subseteq A$ for any positive integer $m$. So for any fixed $a \in A$, we have $w_{i}(h)^{m} a \in A$ and hence $w_{i}(h)^{m} \in a^{-1} A$. Since $A$ is finitely generated, so is $a^{-1} A$. Thus $D\left[w_{i}(h)\right] \subseteq a^{-1} A$ is a finitely generated $D$-module, since $D$ is Noetherian. Hence by Zariski-Samuel (1958, p. 254) $w_{i}(h) \in F$ is integral over $D$, that is, $w_{i}(h) \in \bar{D}$. 
We are now in position to characterize integral orders in $L$ which contain $L_{D}$. First recall that by Chevalley (1961), Kostant (1966) or Steinberg (1968, pp. 7-21), for any representation $\pi: L \rightarrow$ End $V$ where $V$ is a finite dimensional vector space over $F$, there is a lattice $\mathscr{L}$ in $V$ invariant under all $\pi\left(e_{r}^{m} / m !\right)$ for $e_{r} \in B$ and $m$ a positive integer. In particular $\mathscr{L}$ is invariant under all $\pi\left(e_{r}\right)$ and hence under all $\pi\left(h_{r}\right)=\pi\left(\left[e_{r}, e_{-r}\right]\right)$. Thus $\mathscr{L}$ is $\pi\left(L_{D}\right)$-invariant. Hence we see that $L_{D}$ is integral in the sense of Definition 6. We can say considerably more.

Theorem 4. Suppose that $D$ is a Noetherian integral domain of characteristic 0 , and $L$ is of rank at least 2 . If $\mathcal{O} L_{D}$ is an integral order, then $\mathcal{O} \subseteq L_{\bar{D}}$, provided that in case $L$ is of type $C_{n}, 2$ is invertible in $D$.

Proof. Let $x=h+\sum c_{r} e_{r} \in \mathcal{O}$. Then using $e_{r} \in \mathcal{O}$ as multipliers, we obtain as in Theorem 2 every $c_{r} e_{r} \in \mathcal{O}$. Then $\left[c_{r} e_{r}, e_{-r}\right]=c_{r} h_{r} \in \mathcal{O}$. So $c_{r} h_{r}$ is an integral element, since $\mathcal{O}$ is integral. Then by Lemma $5, c_{r} h_{r} \in \bar{D} P^{\perp}$. Then by Lemma 2 , $c_{r} \in \bar{D}$ for each root $r$. Then $x-\sum c_{r} e_{r}=h \in \mathcal{O}$ and so $h$ is an integral element. Hence by Lemma $5, h \in \bar{D}_{\bar{D}} P^{\perp}=H_{\bar{D}}$ and hence $x \in L_{\bar{D}}$.

COROLLARY. If $L$ has rank at least $2, D$ is a Dedekind domain, and the hypotheses of Theorem 4 hold, then $\mathcal{O}=L_{D}$. Thus in this case $L_{D}$ is a maximal integral order in $L$.

Proof. In this case $D=\bar{D}$ since $D$ is integrally closed, so we have $L_{D} \subseteq \mathcal{O} \subseteq L_{D}$.

In particular if $D$ is the ring of algebraic integers in a finite algebraic extension $K$ of $Q$ we get Theorem 2 of Hyman (1966) in the non-symplectic cases, and the full result in all cases if $2 \in D$ is invertible.

We can extend Theorem 4 in a weakened form to the case of prime characteristic.

Definition 7. Suppose that $L_{F}=F \otimes_{\mathbf{Z}} L_{\mathbf{Z}}$ where $L$ is simple over the complex field. If $D$ is of characteristic $p$ with field of fractions $F$, then $O \subseteq L$ will be called a partially integral order in case $\mathcal{O}$ is $\sigma$-integral for every representation $\sigma$ of $L_{F}$ obtainable from a complex representation $\pi$ of $L$ by reduction modulo $p$. An element $x \in L_{F}$ is a partially integral element if it is $\sigma$-integral for every such $\sigma$.

Thus $L_{D}$ is a partially integral order. The reasoning of Lemma 5 establishes the following result immediately.

Lemma 6. If $h \in H$ is partially integral and $D$ is Noetherian, then $w_{i}(h) \in \bar{D}$ for every fundamental weight $w_{i}$ and so $h \in H_{\bar{D}}=\bar{D}_{\bar{D}} P^{\perp}$.

The foregoing now enables us to carry over verbatim the proof of Theorem 4 (with "partially" prefixed to each occurrence of "integral") to establish the following, which must be termed a partial result. 
Theorem 5. Let $L, D, F$, and $\mathcal{O}$ be as in Definition 7 with $L$ of rank at least 2. If $D$ is Noetherian and $\mathcal{O} \supseteq L_{D}$ is a partially integral order, then $\mathcal{O} \subseteq L_{\bar{D}}$ provided that $2 \in D$ is invertible in case $L$ is of type $C_{n}$. In particular, if $D$ is a Dedekind domain, then $L_{D}$ is a maximal partially integral order.

\section{Acknowledgements}

The author is indebted to Professor George B. Seligman for calling his attention to the existence of Hyman's dissertation and suggesting the possible relevance of our paper (1969) and Stewart (1973) to it. The author is also indebted to Professors William J. Wickless and Joseph B. Dennin, Jr., for helpful conversations.

\section{References}

E. Abe (1969), "Chevalley groups over local rings", Tohoku J. Math. 21, 474-494.

E. Abe and K. Suzuki (1976), "On normal subgroups of Chevalley groups over commutative rings", Tohoku J. Math. 28, 185-198.

C. Chevalley (1955), "Sur certains groupes simples", Tohoku J. Math. 7, 14-66.

C. Chevalley (1961), Séminaire Bourbaki, Fasc. 1, Exp. 219 (Secrétariat Mathématique, Paris).

C. W. Curtis and I. Reiner (1962), Representation Theory of Finite Groups and Associative Algebras (Wiley-Interscience, New York).

J. F. Hurley (1969), "Ideals in Chevalley algebras", Trans. Amer. Math. Soc. 137, 245-258.

J. F. Hurley (1971), "Extensions of Chevalley algebras", Duke Math. J. 38, 349-356.

J. F. Hurley (1971), "Some normal subgroups of elementary subgroups of Chevalley groups over rings", American J. Math. 93, 1059-1069.

M. H. Hyman (1966), "Some aspects of the arithmetic theory of Lie algebras", Ph.D. Dissertation, Yale University, New Haven, Conn.

N. Jacobson (1953), Lectures in Abstract Algebra, Vol. II (Van Nostrand, Princeton, N.J.).

N. Jacobson (1962), Lie Algebras (Wiley-Interscience, New York).

B. Kostant (1966), "Groups over Z", Proc. Symposia Pure Math. 9, 90-98.

G. B. Seligman (1967), Modular Lie Algebras (Springer-Verlag, New York, Heidelberg and Berlin).

T. A. Springer (1966), "Some arithmetical results on semisimple Lie algebras", Inst. Hautes Études Sci. Publ. Math. 30, 475-501.

R. Steinberg (1965), "Regular elements of semisimple algebraic groups", Inst. Hautes Études Sci. Publ. Math. 25, 49-80.

R. Steinberg (1968), Lectures on Chevalley Groups (mimeographed, Yale Univ. Math. Dept., New Haven, Conn.).

I. Stewart (1973), "Central simplicity and Chevalley algebras", Compositio Math. 26, 111-118.

O. Zariski and P. Samuel (1958), Commutative Algebra, Vol. I (Van Nostrand, Princeton, N.J.).

Department of Mathematics

Research School of Physical Sciences

The Australian National University

Canberra, A.C.T.

Current postal address:

Department of Mathematics

The University of Connecticut

Storrs, Connecticut 06268 